\title{
Conocimientos, actitudes y prácticas preventivas frente a segunda ola del COVID-19, La Libertad- Perú
}

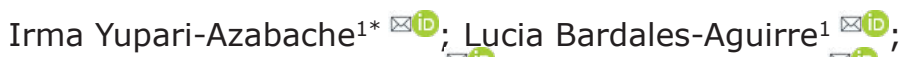 \\ Shamir Barros-Sevillano ${ }^{1,2} \bowtie\left(\mathbb{0} ;\right.$; Jorge Díaz-Ortega ${ }^{1} \bowtie(0)$.
}

\begin{abstract}
${ }^{1}$ Universidad César Vallejo, Grupo de Investigación en Enfermedades Infecciosas y Transmisibles. Trujillo, Perú. ${ }^{2}$ Sociedad Científica de Estudiantes de Medicina de la Universidad César Vallejo. Trujillo, Perú.

*Correspondencia: iyupari@ucv.edu.pe
\end{abstract}

Recibido: Junio 2021; Aceptado: Noviembre 2021; Publicado: Diciembre 2021.

\section{RESUMEN}

Objetivo. Analizar los conocimientos, actitudes y prácticas preventivas frente a la segunda ola del COVID-19 en residentes de La Libertad- Perú. Material y métodos. Se aplicó un diseño transversal, correlacional; se utilizó un cuestionario debidamente validado y los datos fueron recolectados usando un formulario virtual a 294 residentes del departamento de La Libertad, cuya muestra fue hallada cumpliendo los parámetros estadísticos requeridos. Resultados. Las características de los encuestados fueron edad promedio de $28.3 \pm 9.9$ años y $71.8 \%$ mujeres. Las personas con nivel de conocimiento bueno y actitud adecuada tenían características como estado civil casado o conviviente, tenían hijos, grado de instrucción superior y contaban con trabajo estable. Las variables edad y sexo son las que se asociaron al nivel de actitud, así como el hecho de conocer las formas de contagio se asoció significativamente con las actitudes y practicas preventivas que se tiene frente a la segunda ola del COVID-19, por último, un $38.1 \%$ de personas encuestadas indicaron que no confían en la efectividad de la vacuna. Conclusiones. Los residentes encuestados del departamento de la LibertadPerú presentan buenos niveles de conocimiento, actitudes y practicas preventivas, sin embargo, se apreció que no tienen muy claro las formas de contagio y esto sumado a una mala economía, ha llevado al incremento de casos.

Palabras clave: Actitudes; prácticas; COVID-19 (Fuente: MeSH).

\section{ABSTRACT}

Objective. To analyze the knowledge, attitudes and preventive practices regarding the second wave of COVID-19 in residents of La Libertad, Peru. Materials and methods. A cross-sectional, correlational design was applied; a duly validated questionnaire was used and data were collected using a virtual form from 294 inhabitants of the department of La Libertad, whose sample was found to meet the required statistical parameters. Results. The characteristics of the respondents were average age of $28.3 \pm 9.9$ years and $71.8 \%$ women. People with a good level of knowledge and an adequate attitude had characteristics such as married or cohabiting marital status, had children, a higher level of education and had stable work. The variables age and sex are those that were

Como citar (Vancouver).

Yupari-Azabache I, Bardales-Aguirre L, Barros-Sevillano S, Díaz-Ortega J. Conocimientos, actitudes y prácticas preventivas frente a segunda ola del CoviD-19, La Libertad- Perú. Rev MVZ Córdoba. 2022; 27(1):e2467. https://doi.org/10.21897/rmvz.2467 
associated with the level of attitude, as well as the fact of knowing the forms of contagion, which was significantly associated with the attitudes and preventive practices of the second wave of COVID-19 Finally, $38.1 \%$ of people surveyed indicated that they do not trust the effectiveness of the vaccine Conclusions. Residents presented good levels of knowledge, attitudes and preventive practices. However, it was noted that they are not clear about the ways of contagion. Added to the indifference towards vaccines and the economic crisis, this could have contributed to a significant increase in the number of cases.

Keywords: Attitudes; practice; COVID-19 (Source: MeSH).

\section{INTRODUCCION}

En marzo del 2020 se detectó al primer paciente con COVID-19 en el Perú. El gobierno peruano decretó estado de emergencia desde el 15 de marzo, pero a pesar de ello los contagiados fueron aumentando de tal forma que los hospitales colapsaron en una primera ola, teniendo sus picos de muertes más altas en julio y agosto. Poco a poco se ha ido reactivando la economía de manera progresiva, según lo establecido por el gobierno, sin embargo, llegado el mes de noviembre del 2020, al ver que hubo una disminución de casos, sumado a la llegada de las fiestas navideñas con el verano, los residentes empezaron a descuidarse de las medidas de prevención. A partir del mes de febrero inició la segunda ola que conllevó a una mayor morbimortalidad, mayor colapso de pacientes con COVID-19 y escasez de oxígeno medicinal en los centros hospitalarios $(1,2)$.

El Perú, representó en su momento, uno de los epicentros de esta enfermedad, tal es así que, a la quincena de mayo del 2021 , se acercó a los 2 millones de casos confirmados, y cerca de 70 mil muertos oficiales por COVID-19. (1). La Libertad es un departamento del Perú, situado al norte del país y es uno de los más afectados con la segunda ola de contagios, tal es así que se ha registrado una letalidad de $5.49 \%$, segunda tasa más alta de todos los departamentos del Perú (3).

Una proporción elevada de pacientes llegan a tener neumonía bilateral, y en muchos casos esto conlleva a la necesidad de soporte respiratorio. Se estima que un $30 \%$ de pacientes con esta enfermedad requieren ingreso hospitalario, y del 5 al 10\% cuidados intensivos. Así también se estima que la letalidad por COVID-19 varía del $2 \%$ a más del $10 \%$ en algunos países del mundo, siendo los más afectados los adultos mayores con comorbilidades (4).
Por una cuestión natural los virus se reproducen produciendo copias de sí mismos con la participación de sus propias enzimas expresadas por su genoma y de la célula huésped (5). Las mutaciones que más preocupan para el ámbito científico son las modificaciones que se producen en la proteína de la espiga, en específico la conocida como E484K con una mayor afinidad de unión a las células humanas encontrada en las variantes sudafricana (B1351), brasileña (P1) y británica (B117), lo que permite un interés en saber si las mutaciones en la glicoproteína de la espiga median para escapar de los anticuerpos del huésped y comprometer la eficacia de la vacuna, debido a que esta proteína es el principal antígeno viral en las vacunas desarrolladas hasta el momento, como que también podría aumentar la transmisibilidad en nuevas olas de infección (6).

El 7 de febrero del 2021 llegó el primer lote de vacunas al Perú, iniciándose el proceso de inmunización contra la COVID-19, sin embargo, al igual que en el resto del mundo, en Perú, la aceptación de la vacuna no es total, debido principalmente al miedo a los efectos adversos que esta puede producir (7).

El cumplimiento de las restricciones dadas por el gobierno es esencial, sin embargo, la economía informal y la pobreza monetaria son factores que afectan este cumplimiento, así también la indiferencia de las personas en su mayoría jóvenes tal es así que en muchos operativos realizados por las autoridades se encontraron residentes incumpliendo normas dispuestas por el gobierno $(1,3)$.

La difusión de medidas para disminuir la propagación de esta enfermedad considera una baja interacción social y adopción de conductas de prevención como el lavado de manos y uso adecuado de la mascarilla. Investigaciones anteriores afirman que el mantener distanciamiento físico se asocia 
fuertemente a la protección frente al coronavirus; y sobre todo con la llegada de las mutaciones, la protección ocular o el uso de las máscaras faciales pueden ofrecer beneficios adicionales de protección $(8,9)$.

Por todo lo expuesto nos hemos interesado en el desarrollo de una nueva investigación teniendo como objetivo general analizar los conocimientos, actitudes y prácticas preventivas frente a la segunda ola del COVID-19 en residentes de La Libertad- Perú. Como objetivos específicos determinar si existe asociación entre las características como edad, sexo, estado civil, grado de instrucción, si tiene hijos y un trabajo estable con los conocimientos, actitudes y prácticas preventivas frente a la segunda ola del COVID-19, determinar si existe asociación entre los niveles de conocimiento y sus dimensiones con las actitudes y prácticas preventivas frente a la segunda ola del COVID-19 e identificar las actitudes y practicas preventivas más comunes en residentes de La Libertad- Perú.

\section{MATERIALES Y MÉTODOS}

Tipo de estudio, población y muestra. La presente investigación tiene un enfoque cuantitativo, de tipo básico, y diseño transversal correlacional (10).

La población estuvo conformada por todos los residentes del departamento de La Libertad con acceso a internet, que tienen entre 18 a 70 años; las encuestas se realizaron online debido al estado de emergencia sanitaria. Los datos se recolectaron entre el 15 de marzo al 30 de abril. En este contexto debido a la accesibilidad, se utilizó un muestreo por conveniencia (no probabilístico), logrando una muestra de 294 residentes del departamento de La Libertad. Para el cálculo de esta muestra, se consideró 95\% de confiabilidad, 5\% de error ajustado y una prevalencia de $73.5 \%$, que corresponde al porcentaje obtenido para un buen nivel de conocimiento del COVID-19 en los encuestados de un estudio realizado anteriormente $(10,11)$.

Recolección de datos. Se empleó una encuesta como técnica y un cuestionario como instrumento, el cual se elaboró utilizando formulario Google, siendo aplicado de manera virtual a la muestra seleccionada, realizando difusión por medio de las redes sociales (WhatsApp, Facebook) y correo electrónico. Este cuestionario fue extraído de un estudio realizado en el año 2020 en Trujillo, departamento de La Libertad, Perú $(10,11)$.

Se hicieron algunas modificaciones al cuestionario considerando que en el tiempo que ha pasado se han realizado algunos descubrimientos con respecto a la enfermedad del COVID-19, por lo que fue nuevamente validado por cinco profesionales: 4 profesionales del área de salud ( 2 médicos, 1 microbiólogo, 1 biólogo) y un metodólogo, obteniendo una validación del $99 \%$ con el estadístico de V-Aiken para el cuestionario de conocimientos, del $100 \%$ para el cuestionario de actitudes y $100 \%$ para prácticas.

El cuestionario incluye preguntas de características de los encuestados como edad, sexo, estado civil, el tener hijos, grado de instrucción, contar con trabajo estable y lugar de residencia. Seguido de ello tiene 67 preguntas de las cuales 33 miden conocimiento general, dividiéndose en dimensiones, donde 10 preguntas miden conocimiento de síntomas, 8 para formas de contagio, 8 para acciones a tomar y 7 de conocimiento de la enfermedad; para medir las actitudes preventivas se usaron 10 preguntas y para medir prácticas preventivas 18 preguntas (11).

Para medir el conocimiento se utilizó la escala dicotómica considerando a la respuesta buena con la calificación de 1 y a la respuesta mala con 0 , otorgándole el nivel de calificación de mala cuando se obtuvo de 0 a 11 puntos, regular de 12 a 24 puntos y buena de 25 a 34 puntos. En el cuestionario de actitud se trabajó con escala de Likert considerando puntajes desde 5: completamente de acuerdo, 4: de acuerdo, 3: no sabe, no opina, 2: en desacuerdo y 1 : completamente en desacuerdo, con 3 preguntas en escala inversa; obteniendo una calificación final de inadecuada de 9 a 33 puntos y adecuada de 34 a 45 puntos. Por último, en el cuestionario de prácticas también se utilizó escala de Likert calificándola con 5: siempre, 4: frecuentemente, 3: ocasionalmente, 2: rara vez y 1: nunca; considerándose una práctica inadecuada de 18 a 65 puntos y adecuada de 66 a 91 puntos. Se considera actitud adecuada cuando la persona adopta una disposición para prevenir el riesgo de infección de COVID-19, teniendo posturas favorables, y la práctica adecuada se manifiesta con los comportamientos que realiza para evitar el contagio y la propagación del virus (11).

Se volvió a medir la fiabilidad del instrumento, obteniendo en el nivel de conocimiento general 
una confiabilidad de Kuder Richardson de 0.77 que indica un nivel muy aceptable, y analizando las dimensiones para conocimiento de síntomas resultó 0.79 que es una fiabilidad muy aceptable, para formas de contagio se obtuvo 0.71 , para conocimiento de la enfermedad resultó 0.71 también, indicando una fiabilidad aceptable y para acciones a tomar se obtuvo 0.77 , mostrando una muy aceptable fiabilidad. La siguiente parte del instrumento evaluó actitudes preventivas en la segunda ola del COVID-19, las cuales fueron evaluadas por alfa de Cronbach obteniendo un nivel aceptable con 0.74. En la última parte del cuestionario se midieron las prácticas preventivas frente a la segunda ola del COVID-19, la fiabilidad de estas preguntas resultó de 0.97 que corresponde a un nivel excelente (12).

Análisis de resultados. Se utilizó primero el programa Excel para la elaboración de la base, este luego se exportó al programa SPSS versión 27. Se aplicó un análisis descriptivo y el test estadístico Chi cuadrado para determinar cuáles de las características analizadas se encontraban asociadas al conocimiento, actitudes y prácticas preventivas frente a la segunda ola del COVID-19. La aplicación de la Chi cuadrado requiere una condición importante en la tabla (de los valores esperados de las celdas, por lo menos el $80 \%$ deben ser mayores que 5 ), en las situaciones que no se cumple con ello, se hizo uso de la Tau B de Kendal que representa una medida de asociación para variables cualitativas, y en el caso de la variable sexo se agregó el cálculo del OR para medir el riesgo $(13,14)$.

Aspectos éticos. Este estudio se encuentra aprobado por comité de ética en la Universidad César Vallejo (Informe 006-CE-FCS-UCV-21), y además cada participante brindó su consentimiento informado antes de la aplicación del cuestionario.

\section{RESULTADOS}

La edad promedio de los 294 residentes, fue de $28.3 \pm 9.9$ años, el $71.8 \%$ eran mujeres, el $74.8 \%$ eran solteros, el $68.7 \%$ no tenían hijos, el $92.2 \%$ eran de nivel superior y sobre el entorno laboral el $64.3 \%$ de los participantes no tenían trabajo estable (Tabla 1).
Tabla 1. Características de los residentes encuestados de La Libertad.

\begin{tabular}{|c|c|c|c|}
\hline \multicolumn{2}{|c|}{ Características } & Frecuencia & Porcentaje \\
\hline \multicolumn{2}{|c|}{ Edad (Media / Desv. Estándar) } & \multicolumn{2}{|c|}{$28.3 \pm 9.9$ años } \\
\hline \multirow{3}{*}{ Sexo } & Masculino & 83 & $28.2 \%$ \\
\hline & Femenino & 211 & $71.8 \%$ \\
\hline & Soltero & 220 & $74.8 \%$ \\
\hline Estado Civil & $\begin{array}{c}\text { Casado o } \\
\text { conviviente }\end{array}$ & 74 & $25.2 \%$ \\
\hline \multirow{2}{*}{ Tiene hijos } & No & 202 & $68.7 \%$ \\
\hline & $\mathrm{Si}$ & 92 & $31.3 \%$ \\
\hline \multirow{2}{*}{$\begin{array}{l}\text { Grado de } \\
\text { instrucción }\end{array}$} & $\begin{array}{l}\text { Primaria o } \\
\text { secundaria }\end{array}$ & 23 & $7.8 \%$ \\
\hline & Superior & 271 & $92.2 \%$ \\
\hline \multirow{2}{*}{ Trabajo Estable } & No & 189 & $64.3 \%$ \\
\hline & $\mathrm{Si}$ & 105 & $35.7 \%$ \\
\hline \multicolumn{2}{|c|}{ Total } & 294 & $100.0 \%$ \\
\hline
\end{tabular}

Se realizó un análisis con porcentajes tomados por fila, notando que los participantes con edades entre 30 a mas, ser mujer, de estado civil casado o conviviente, el hecho de tener hijos, tener grado de instrucción superior y contar con trabajo estable son las características de las personas con mayor nivel de conocimiento bueno y actitud adecuada. Así también se puede apreciar que las variables edad y sexo son las que se encuentran asociadas al nivel de actitud $(p<0.05)$. En el caso de la edad se podría afirmar que la asociación es positiva y el hecho de tener mayor edad conlleva a tener un conocimiento bueno y una actitud adecuada, así como podemos deducir que ser hombre es de mayor riesgo para tener un conocimiento regular y actitud inadecuada (Tablas 2 y 3 ).

Con similar análisis que las anteriores tablas, se notó que las características en los participantes con mayor porcentaje en prácticas adecuadas son del grupo de mayores de 60 años, de estado civil casado o conviviente, el hecho de tener hijos, tener grado de instrucción superior y contar con trabajo estable, sin embargo, en este caso ninguna de las variables analizadas se asoció a las prácticas preventivas adecuadas (Tabla 4). 
Yupari-Azabache et al - Conocimientos, actitudes y prácticas preventivas del COVID-19

Tabla 2. Características asociadas al nivel de conocimiento en la segunda ola del COVID -19 en residentes de La Libertad.

\begin{tabular}{|c|c|c|c|c|c|c|c|}
\hline \multicolumn{2}{|c|}{ Características } & \multirow{2}{*}{$\begin{array}{c}\text { Regular } \\
47\end{array}$} & \multirow{2}{*}{$\begin{array}{c}\% \\
24.2 \%\end{array}$} & \multirow{2}{*}{$\begin{array}{c}\text { Bueno } \\
147\end{array}$} & \multirow{2}{*}{$\begin{array}{c}\text { \% } \\
75.8 \%\end{array}$} & \multirow[t]{2}{*}{ Chi Cuadrado } & \multirow[t]{2}{*}{ Sig. } \\
\hline & $18-29$ años & & & & & & \\
\hline \multirow[t]{2}{*}{ Edad } & 30 - 59 años & 14 & $14.6 \%$ & 82 & $85.4 \%$ & $0.10^{a}$ & 0.06 \\
\hline & 60 a más años & 1 & $25.0 \%$ & 3 & $75.0 \%$ & & \\
\hline \multirow{2}{*}{ Sexo } & Masculino & 24 & $28.9 \%$ & 59 & $71.1 \%$ & \multirow{2}{*}{$4.26^{b}$} & \multirow{2}{*}{0.04} \\
\hline & Femenino & 38 & $18.0 \%$ & 173 & $82.0 \%$ & & \\
\hline \multirow{2}{*}{ Estado Civil } & Soltero & 50 & $22.7 \%$ & 170 & $77.3 \%$ & \multirow{2}{*}{1.41} & \multirow{2}{*}{0.24} \\
\hline & Casado o conviviente & 12 & $16.2 \%$ & 62 & $83.8 \%$ & & \\
\hline \multirow{2}{*}{ Tiene hijos } & No & 46 & $22.8 \%$ & 156 & $77.2 \%$ & \multirow{2}{*}{1.10} & \multirow{2}{*}{0.29} \\
\hline & $\mathrm{Si}$ & 16 & $17.4 \%$ & 76 & $82.6 \%$ & & \\
\hline \multirow{2}{*}{ Grado de Instrucción } & Primaria o secundaria & 6 & $26.1 \%$ & 17 & $73.9 \%$ & \multirow{2}{*}{0.37} & \multirow{2}{*}{0.54} \\
\hline & Superior & 56 & $20.7 \%$ & 215 & $79.3 \%$ & & \\
\hline \multirow{2}{*}{ Cuenta con trabajo estable } & No & 44 & $23.3 \%$ & 145 & $76.7 \%$ & \multirow{2}{*}{1.53} & \multirow{2}{*}{1.22} \\
\hline & $\mathrm{Si}$ & 18 & $17.1 \%$ & 87 & $82.9 \%$ & & \\
\hline
\end{tabular}

Nota: (a) Se uso Tau B de Kendal, (b): OR: 2(I.C.95\%: 1.03-3.34)

Tabla 3. Características asociadas a las actitudes en la segunda ola del COVID-19 en residentes de La Libertad.

\begin{tabular}{|c|c|c|c|c|c|c|c|}
\hline \multirow{2}{*}{\multicolumn{2}{|c|}{ Características }} & \multicolumn{4}{|c|}{ ACTITUD } & \multirow{2}{*}{ Chi Cuadrado } & \multirow{2}{*}{ Sig. } \\
\hline & & Inadecuada & $\%$ & Adecuada & $\%$ & & \\
\hline \multirow{3}{*}{ Edad } & 18 - 29 años & 21 & $10.8 \%$ & 173 & $89.2 \%$ & \multirow{3}{*}{$0.12^{\mathrm{a}}$} & \multirow{3}{*}{0.02} \\
\hline & $30-59$ años & 4 & $4.2 \%$ & 92 & $95.8 \%$ & & \\
\hline & 60 a más años & 0 & $0.0 \%$ & 4 & $100.0 \%$ & & \\
\hline \multirow{2}{*}{ Sexo } & Masculino & 12 & $14.5 \%$ & 71 & $85.5 \%$ & \multirow{2}{*}{$5.27^{b}$} & \multirow{2}{*}{0.022} \\
\hline & Femenino & 13 & $6.2 \%$ & 198 & $93.8 \%$ & & \\
\hline \multirow[b]{2}{*}{ Estado Civil } & Soltero & 19 & $8.6 \%$ & 201 & $91.4 \%$ & \multirow[b]{2}{*}{0.02} & \multirow[b]{2}{*}{0.88} \\
\hline & $\begin{array}{l}\text { Casado o } \\
\text { conviviente }\end{array}$ & 6 & $8.1 \%$ & 68 & $91.9 \%$ & & \\
\hline \multirow{2}{*}{ Tiene hijos } & No & 18 & $8.9 \%$ & 184 & $91.1 \%$ & \multirow{2}{*}{0.14} & \multirow{2}{*}{0.71} \\
\hline & $\mathrm{Si}$ & 7 & $7.6 \%$ & 85 & $92.4 \%$ & & \\
\hline \multirow{2}{*}{ Grado de Instrucción } & $\begin{array}{l}\text { Primaria o } \\
\text { secundaria }\end{array}$ & 3 & $13.0 \%$ & 20 & $87.0 \%$ & \multirow{2}{*}{0.18} & \multirow{2}{*}{0.67} \\
\hline & Superior & 22 & $8.1 \%$ & 249 & $91.9 \%$ & & \\
\hline \multirow{2}{*}{ Cuenta con trabajo estable } & No & 17 & $9.0 \%$ & 172 & $91.0 \%$ & \multirow{2}{*}{0.16} & \multirow{2}{*}{0.69} \\
\hline & $\mathrm{Si}$ & 8 & $7.6 \%$ & 97 & $92.4 \%$ & & \\
\hline
\end{tabular}

Nota: (a) Se usó Tau B de Kendal, (b): OR:3 (I.C.95\%: 1.12-5.90)

Tabla 4. Características asociadas a las practicas preventivas frente a la segunda ola del COVID-19 en residentes de La Libertad.

\begin{tabular}{|c|c|c|c|c|c|c|c|}
\hline \multirow{2}{*}{\multicolumn{2}{|c|}{ Características }} & \multicolumn{4}{|c|}{ Practicas preventivas } & \multirow{2}{*}{ Chi Cuadrado } & \multirow{2}{*}{ Sig. } \\
\hline & & Inadecuada & $\%$ & Adecuada & $\%$ & & \\
\hline \multirow{3}{*}{ Edad } & 18 - 29 años & 10 & $5.2 \%$ & 184 & $94.8 \%$ & \multirow{3}{*}{0.07} & \multirow{3}{*}{0.13} \\
\hline & 30 - 59 años & 2 & $2.1 \%$ & 94 & $97.9 \%$ & & \\
\hline & 60 a más años & 0 & $0.0 \%$ & 4 & $100.0 \%$ & & \\
\hline \multirow{2}{*}{ Sexo } & Masculino & 3 & $3.6 \%$ & 80 & $96.4 \%$ & \multirow{2}{*}{0.064} & \multirow{2}{*}{0.8} \\
\hline & Femenino & 9 & $4.3 \%$ & 202 & $95.7 \%$ & & \\
\hline \multirow{2}{*}{ Estado Civil } & Soltero & 11 & $5.0 \%$ & 209 & $95.0 \%$ & \multirow{2}{*}{1.88} & \multirow{2}{*}{0.17} \\
\hline & Casado o conviviente & 1 & $1.4 \%$ & 73 & $98.6 \%$ & & \\
\hline \multirow{2}{*}{ Tiene hijos } & No & 9 & $4.5 \%$ & 193 & $95.5 \%$ & \multirow{2}{*}{0.23} & \multirow{2}{*}{0.63} \\
\hline & $\mathrm{Si}$ & 3 & $3.3 \%$ & 89 & $96.7 \%$ & & \\
\hline \multirow{2}{*}{ Grado de Instrucción } & Primaria o secundaria & 2 & $8.7 \%$ & 21 & $91.3 \%$ & \multirow{2}{*}{0.38} & \multirow{2}{*}{0.54} \\
\hline & Superior & 10 & $3.7 \%$ & 261 & $96.3 \%$ & & \\
\hline \multirow{2}{*}{ Cuenta con trabajo estable } & No & 9 & $4.8 \%$ & 180 & $95.2 \%$ & \multirow{2}{*}{0.63} & \multirow{2}{*}{0.43} \\
\hline & $\mathrm{Si}$ & 3 & $2.9 \%$ & 102 & $97.1 \%$ & & \\
\hline
\end{tabular}


Para determinar los conocimientos y sus dimensiones asociadas con las actitudes y las practicas preventivas de los participantes frente a la segunda ola del coronavirus se ha utilizado el estadístico Tau B de Kendal, obteniéndose que el conocimiento en sus formas de contagio es la única que se asocia significativamente a las actitudes y practicas preventivas que se tiene frente a la segunda ola del COVID-19 $(p<0.05)$ sin embargo, también se observa que el nivel de asociación es muy bajo. Así mismo, se aprecia que en la dimensión formas de contagio la mayoría de las personas muestran un conocimiento regular y a pesar de que en las otras dimensiones tienen un conocimiento bueno, no se observa que esto se asocie con sus actitudes siendo estas también en su mayoría adecuadas (Tablas 5 y 6 ).

Tabla 5. Conocimiento y sus dimensiones asociadas a las actitudes frente a la segunda ola del COVID-19 en residentes de La Libertad.

\begin{tabular}{|c|c|c|c|c|c|c|c|c|c|}
\hline \multirow{2}{*}{\multicolumn{2}{|c|}{ Conocimiento y sus dimensiones }} & \multicolumn{6}{|c|}{ Actitud frente al Coronavirus } & \multirow{2}{*}{$\begin{array}{c}\text { Tau B } \\
\text { Kendal }\end{array}$} & \multirow{2}{*}{ Sig } \\
\hline & & Inadec. & $\%$ & Adec. & $\%$ & Total & $\%$ & & \\
\hline \multirow{3}{*}{$\begin{array}{l}\text { Conocimiento de síntomas de } \\
\text { la infección por Coronavirus }\end{array}$} & Malo & 4 & $1.4 \%$ & 30 & $10.2 \%$ & 34 & $12 \%$ & \multirow{3}{*}{0.03} & \multirow{3}{*}{0.65} \\
\hline & Regular & 6 & $2.0 \%$ & 68 & $23.1 \%$ & 74 & $25 \%$ & & \\
\hline & Bueno & 15 & $5.1 \%$ & 171 & $58.2 \%$ & 186 & $63 \%$ & & \\
\hline \multirow{3}{*}{$\begin{array}{l}\text { Conocimiento de formas de } \\
\text { contagio }\end{array}$} & Malo & 1 & $0.3 \%$ & 6 & $2.0 \%$ & 7 & $2 \%$ & \multirow{3}{*}{0.11} & \multirow{3}{*}{0.04} \\
\hline & Regular & 20 & $6.8 \%$ & 171 & $58.2 \%$ & 191 & $65 \%$ & & \\
\hline & Bueno & 4 & $1.4 \%$ & 92 & $31.3 \%$ & 96 & $33 \%$ & & \\
\hline \multirow{3}{*}{$\begin{array}{c}\text { Conocimiento sobre la } \\
\text { enfermedad }\end{array}$} & Malo & 1 & $0.3 \%$ & 0 & $0.0 \%$ & 1 & $0 \%$ & \multirow{3}{*}{0.01} & \multirow{3}{*}{0.84} \\
\hline & Regular & 9 & $3.1 \%$ & 106 & $36.1 \%$ & 115 & $39 \%$ & & \\
\hline & Bueno & 15 & $5.1 \%$ & 163 & $55.4 \%$ & 178 & $61 \%$ & & \\
\hline \multirow{3}{*}{$\begin{array}{l}\text { Conocimiento de acciones a } \\
\text { tomar en caso de infectarse }\end{array}$} & Malo & 1 & $0.3 \%$ & 2 & $0.7 \%$ & 3 & $1 \%$ & \multirow{3}{*}{0.081} & \multirow{3}{*}{0.2} \\
\hline & Regular & 11 & $3.7 \%$ & 92 & $31.3 \%$ & 103 & $35 \%$ & & \\
\hline & Bueno & 13 & $4.4 \%$ & 175 & $59.5 \%$ & 188 & $64 \%$ & & \\
\hline \multirow{2}{*}{ Conocimiento General } & Regular & 8 & $2.7 \%$ & 54 & $18.4 \%$ & 62 & $21.1 \%$ & \multirow{2}{*}{1.96} & \multirow{2}{*}{0.16} \\
\hline & Bueno & 17 & $5.8 \%$ & 215 & $73.1 \%$ & 232 & $78.9 \%$ & & \\
\hline Total & & 25 & $8.5 \%$ & 269 & $91.5 \%$ & 294 & $100.0 \%$ & & \\
\hline
\end{tabular}

Tabla 6. Conocimiento y sus dimensiones asociadas a las practicas preventivas frente a la segunda ola del COVID-19 en residentes de La Libertad.

\begin{tabular}{|c|c|c|c|c|c|c|c|c|c|}
\hline \multicolumn{2}{|c|}{ Conocimiento y sus dimensiones } & \multicolumn{6}{|c|}{ Practicas preventivas segunda ola del COVID-19 } & \multirow{2}{*}{$\begin{array}{c}\text { Tau B } \\
\text { Kendal }\end{array}$} & \multirow{2}{*}{ Sig. } \\
\hline & & Inadec. & $\%$ & Adec. & $\%$ & Total & $\%$ & & \\
\hline \multirow{3}{*}{$\begin{array}{l}\text { Conocimiento de Síntomas de la } \\
\text { infección por Coronavirus }\end{array}$} & Malo & 1 & $0.3 \%$ & 33 & $11.2 \%$ & 34 & $11.6 \%$ & \multirow{3}{*}{0.04} & \multirow{3}{*}{0.45} \\
\hline & Regular & 5 & $1.7 \%$ & 69 & $23.5 \%$ & 74 & $25.2 \%$ & & \\
\hline & Bueno & 6 & $2.0 \%$ & 180 & $61.2 \%$ & 186 & $63.3 \%$ & & \\
\hline \multirow{3}{*}{$\begin{array}{l}\text { Conocimiento de formas de } \\
\text { contagio }\end{array}$} & Malo & 1 & $0.3 \%$ & 6 & $2.0 \%$ & 7 & $2.4 \%$ & \multirow{3}{*}{0.12} & \multirow{3}{*}{0.03} \\
\hline & Regular & 10 & $3.4 \%$ & 181 & $61.6 \%$ & 191 & $65.0 \%$ & & \\
\hline & Bueno & 1 & $0.3 \%$ & 95 & $32.3 \%$ & 96 & $32.7 \%$ & & \\
\hline \multirow{3}{*}{$\begin{array}{l}\text { Conocimiento sobre la } \\
\text { enfermedad }\end{array}$} & Malo & 0 & $0.0 \%$ & 1 & $0.3 \%$ & 1 & $0.3 \%$ & \multirow{3}{*}{-0.03} & \multirow{3}{*}{0.6} \\
\hline & Regular & 4 & $1.4 \%$ & 111 & $37.8 \%$ & 115 & $39.1 \%$ & & \\
\hline & Bueno & 8 & $2.7 \%$ & 170 & $57.8 \%$ & 178 & $60.5 \%$ & & \\
\hline \multirow{3}{*}{$\begin{array}{l}\text { Conocimiento de acciones a tomar } \\
\text { en caso de infectarse }\end{array}$} & Malo & 0 & $0.0 \%$ & 3 & $1.0 \%$ & 3 & $1.0 \%$ & \multirow{3}{*}{0.06} & \multirow{3}{*}{0.35} \\
\hline & Regular & 6 & $2.0 \%$ & 97 & $33.0 \%$ & 103 & $35.0 \%$ & & \\
\hline & Bueno & 6 & $2.0 \%$ & 182 & $61.9 \%$ & 188 & $63.9 \%$ & & \\
\hline \multirow{2}{*}{ Conocimiento GENERAL } & Regular & 4 & $1.4 \%$ & 58 & $19.7 \%$ & 62 & $21.1 \%$ & \multirow{2}{*}{0.49} & \multirow{2}{*}{0.48} \\
\hline & Bueno & 8 & $2.7 \%$ & 224 & $76.2 \%$ & 232 & $78.9 \%$ & & \\
\hline Total & & 12 & $4.1 \%$ & 282 & $95.9 \%$ & 294 & $100.0 \%$ & & \\
\hline
\end{tabular}


Se puede apreciar que la mayoría de las personas tienen un nivel de conocimiento bueno y en sus dimensiones, la que figura con mejor nivel por los encuestados, es la de conocimiento de acciones a tomar en caso de infectarse (63.9\%) (Figura 1).

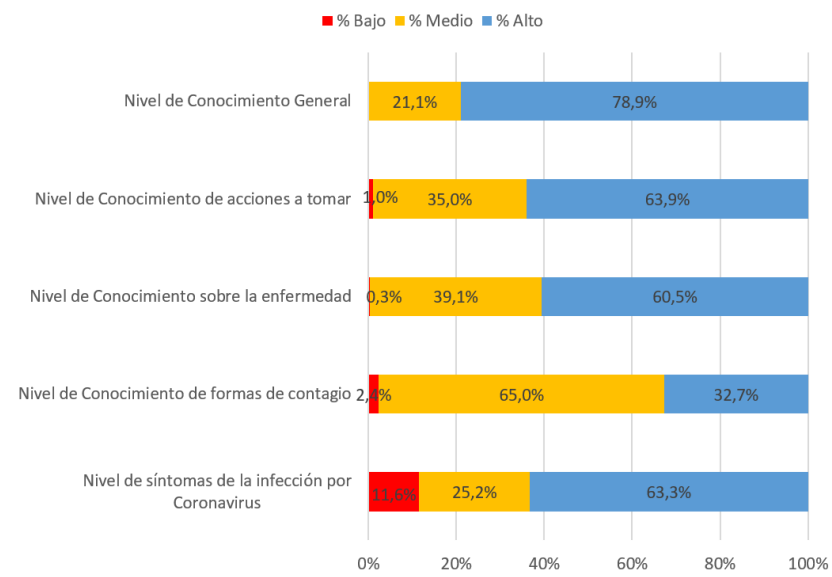

Figura 1. Nivel de conocimiento del COVID 19, según dimensión en residentes de La Libertad.

La mayoría de las personas, es decir el $97.6 \%$ le preocupa que un integrante de la familia pueda contraer esta enfermedad, el $96.3 \%$ explican a su familia como prevenir contagiarse de la enfermedad, sin embargo, en menor proporción, es decir un $61.9 \%$ son personas que confían en la efectividad de las vacunas (Figura 2).

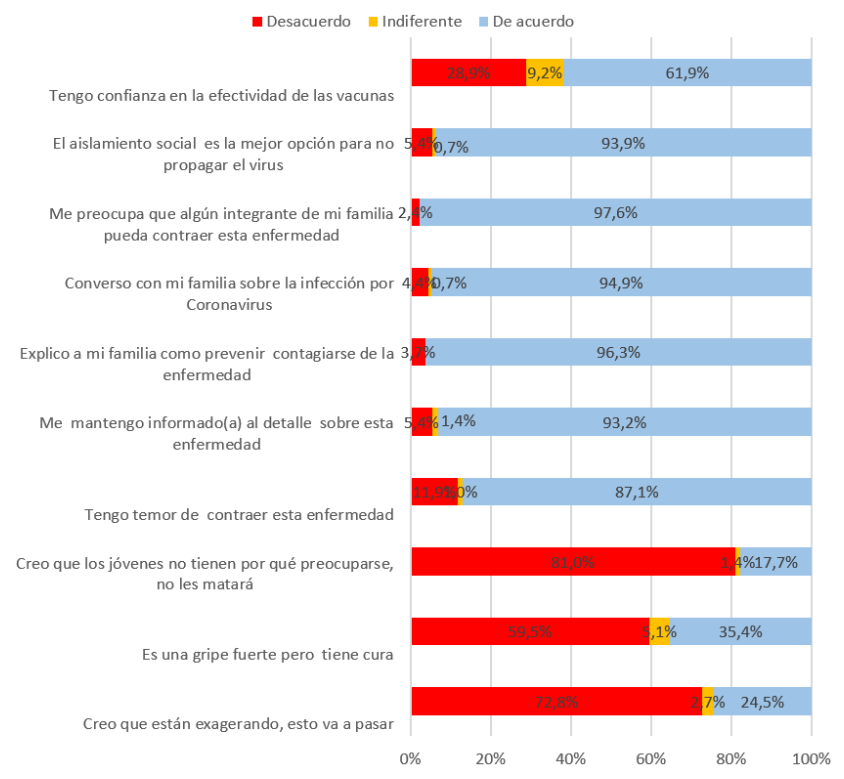

Figura 2. Actitudes de los residentes de La Libertad en la segunda ola del COVID 19.
La mayoría, es decir $98.6 \%$ de personas tienen prácticas adecuadas con respecto a desinfectarse las manos con alcohol, lavarse las manos y respecto al uso de mascarilla, sin embargo, existe también un $76.5 \%$ de personas que consumen comida chatarra (Figura 3).

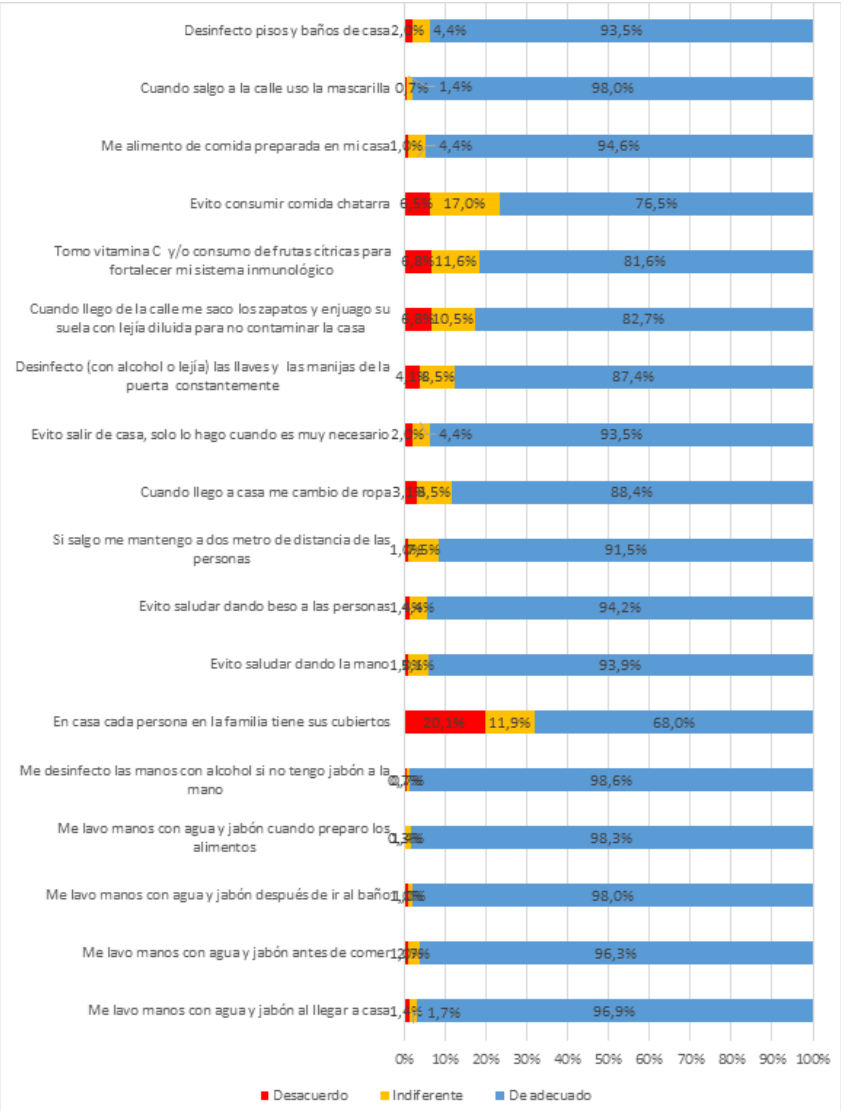

Figura 3. Prácticas preventivas frente a la segunda ola del COVID-19 en residentes de La Libertad.

\section{DISCUSIÓN}

Nuestros resultados demuestran que el nivel de conocimiento es bueno en un $78.9 \%$, las actitudes y prácticas son adecuadas en un $91.5 \%$ y $95.9 \%$ respectivamente, sin embargo, solo la edad y el sexo se encuentran asociadas al nivel de de actitud. En comparación, con otro estudio realizado en Trujillo y a nivel nacional en el año 2020, los resultados en la muestra de la Libertad difieren en cuanto a estas tres variables, encontrándose al sexo masculino como una característica de riesgo para tener una actitud inadecuada y el hecho de tener de 18 a 21 años se asocia a una actitud inadecuada también. Esto se confirma ya que todavía existen actitudes 
como el de pensar que solo es una gripe fuerte $(35.4 \%)$, que se está exagerando (24.5\%) y que los jóvenes no tienen por qué preocuparse porque no los matará $(17.7 \%)(11,15)$.

El conocimiento en sus formas de contagio fue la dimensión que se asoció significativamente a la actitud preventiva que se tiene frente al COVID-19( $p<0.05)$, esto coincide con el estudio hecho en Huánuco- Perú, donde la percepción de desconocimiento de la enfermedad se asoció a las actitudes negativas frente al COVID-19 (16).

A nivel internacional también se han realizado estudios de este tipo, así tenemos en Venezuela, donde los hallazgos demostraron que los participantes tenían un alto nivel de conocimiento sobre COVID-19, sin embargo las personas más jóvenes, con menos educación tienen niveles más bajos de conocimiento, y estos grupos, así como los hombres, tenían menos probabilidades de adoptar prácticas preventivas, similar a nuestros resultados en Perú, característico en sociedades pobres y de informalidad laboral en países latinoamericanos (17).

En Ecuador, los hombres, las personas de 18 a 29 años, las personas solteras y desempleadas tomaron los comportamientos más riesgosos; sus resultados indican que el conocimiento sobre COVID-19 es insuficiente para impulsar un cambio de comportamiento entre los ecuatorianos, por ello el conocimiento igual que en nuestra investigación no tuvo asociación significativa con las prácticas. Así podemos apreciar buenos niveles de conocimiento en nuestros encuestados, pero existen prácticas que siguen siendo inadecuadas como consumir comida chatarra (23.5\%) y no separar los cubiertos por persona en casa $(32 \%)$ de tal forma que es más fácil contagiarse y contagiar a los integrantes la familia (18).

En el estudio realizado en Paraguay la edad promedio de los participantes fue de $29.55 \pm 10.7$ años, similar a nuestro trabajo donde los participantes tenían una edad promedio de

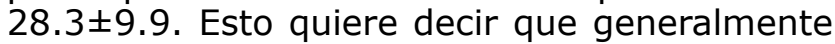
la población joven es la que tiene acceso a desarrollar este tipo de cuestionarios, y se han encontrado similares resultados como conocimientos aceptables, actitudes favorables y las prácticas mayoritariamente adecuadas, así como el hecho de que el género masculino constituye mayor riesgo de prácticas inadecuadas (19), a diferencia del estudio realizado en Camerún donde las mujeres tenían puntuaciones de práctica más bajas en comparación con los hombres (20).
Los hallazgos en nuestro estudio indican que las personas han tomado conciencia en cuanto a las prácticas como el uso de la mascarilla y desinfectarse las manos, similar al estudio realizado en Indonesia y Bangladesh. $(21,22)$. Así también, en Pakistán y Malasia, la mayoría de los encuestados cree que para prevenir la infección con el COVID-19, las personas deben evitar ir a lugares concurridos y practicar higiene adecuada de manos $(23,24)$; sin embargo en nuestro país el no tener un buen conocimiento de las formas de contagio y el hecho de encontrarnos en época electoral ha influido para que las personas no cumplan estas prácticas preventivas dejando de respetar el distanciamiento social asistiendo, a mítines, protestas en contra de una candidatura, dando lugar a que las cifras de contagio aumenten (25).

Otro punto importante a mencionar es que en actitudes a la fecha existe un $38.1 \%$ de personas encuestadas en el departamento de la Libertad que se muestran indiferentes y no confían en la efectividad de la vacuna. En Perú a mayo del 2021 solo se han vacunado al personal de salud y personas de tercera edad, sin embargo, muchas de estas han decidido no colocársela debido a la desinformación periodística en relación a la seguridad y eficacia de las vacunas en diferentes recursos de información (26).

En conclusión, los residentes encuestados del departamento de la Libertad del Perú presentan buenos niveles de conocimiento, actitudes y practicas preventivas, sin embargo, en una de las dimensiones analizadas para el conocimiento se apreció que no tienen muy claro las formas de contagio. La edad y el sexo fueron las únicas variables asociadas al nivel de conocimiento y actitudes; y solo la dimensión formas de contagio se asoció con las actitudes y prácticas. Existe todavía algunas actitudes y prácticas que no son adecuadas en los residentes de La Libertad, esto sumado a una mala economía, ha llevado al incremento de casos.

Esperamos que el estudio facilite la implementación de políticas efectivas por parte de nuestras autoridades, a fin de que la población de La Libertad pueda tomar conciencia y mejore los puntos débiles de sus conocimientos, actitudes y practicas evitando más la propagación de esta pandemia.

\section{Conflicto de intereses}

Los autores declaran que no tienen ningún conflicto de intereses. 


\section{REFERENCIAS}

1. Cabezas C. Pandemia del COVID-19: tormentas y retos. Rev Peru Med Exp Salud Publica. 2020; 37(4):603-4. https://rpmesp. ins.gob.pe/rpmesp/article/view/6866/3976

2. Solari L. El 2021 y sus nuevos retos en el control del SARS-CoV-2. Rev Peru Med Exp Salud Publica. 2021; 38(1):5-6. https://rpmesp.ins.gob.pe/rpmesp/article/ view/7312/4130

3. Ministerio de Salud del Perú [internet] Sala Situacional COVID 19 Perú. Lima: MINSA; 2021. [Citado el 22 de Mayo del 2021] Disponible en: https://covid19.minsa.gob. pe/sala situacional.asp

4. Carrascosa J, Morillas V, Bielsa I, Munera M. Cutaneous Manifestations in the Context of SARS-CoV-2Infection (COVID-19). Actas Dermosifiliogr. 2020; 111(9):734-42. https://doi.org/10.1016/j. adengl.2020.10.001

5. Almubaid Z, Al-Mubaid H. Analysis and comparison of genetic variants and mutations of the novel coronavirus SARS-CoV-2. Gene Rep. 2021; 23:101064. https://doi. org/10.1016/j.genrep.2021.101064

6. Pacheco J. La incógnita del coronavirusVariantes y vacunas - La gestante y su niño. Rev Peru Ginecol Obstet. 2021; 67(1):1-10. https://doi.org/10.31403/rpgo.v67i2311

7. Rzymski P, Zeyland J, Poniedziałek B, Małecka I, Wysocki J. The Perception and Attitudes toward COVID-19 Vaccines: A Cross-Sectional Study in Poland. Vaccines. 2021; 9(4):382. https://doi.org/10.3390/ vaccines 9040382

8. Lehrer S, Rheinstein P. Eyeglasses Reduce Risk of COVID-19 Infection. In vivo. 2021; 35(3):1581-1582. https://doi. org/10.21873/invivo.12414

9. Akhtar J, Garcia AL, Saenz L, Kuravi S, Shu F, Kota K. Can face masks offer protection from airborne sneeze and cough droplets in close-up, face-to-face human interactions?-A quantitative study. Phys Fluids. 2020; 32(12):127112. https://doi. org/10.1063/5.0035072
10. Manterola C, Quiroz G, Salazar P, García N. Metodología de los tipos y diseños de estudio más frecuentemente utilizados en investigación clínica. Rev Med Clin Condes. 2019; 30(1):36-49. https://doi. org/10.1016/j.rmclc.2018.11.005

11. Yupari I, Díaz J, Rodríguez A, Peralta A. Factores asociados a las actitudes y prácticas preventivas frente a la pandemia del COVID-19. Rev MVZ Córdoba. 2020; 25(3):e2052. https://doi.org/10.21897/ rmvz.2052

12. Bolivar E, Villanueva A. Validación y confiabilidad del Cuestionario AQ-27 de actitudes estigmatizadoras hacia pacientes con esquizofrenia en un Hospital General 2015. Rev Neuropsiquiatr. 2017; 80(3):165171. https://doi.org/10.20453/rnp. v80i3.3153

13. Castro M. Bioestadística aplicada en investigación clínica: conceptos básicos. Rev Med Clin Condes. 2019; 30(1):50-65. https:// doi.org/10.1016/j.rmclc.2018.12.002

14. Riffenburgh R, Guillen D. Statistics in Medicine. 4th ed. London: Elsevier; 2020.

15. Ruiz M, Diaz A, Ortiz M. Creencias, conocimientos y actitudes frente a la COVID-19 de pobladores adultos peruanos en cuarentena social Rev Cubana Enferm. 2020; 36:e4251. http://revenfermeria.sld. cu/index.php/enf/article/view/4251

16. Ruiz-Aquino $M$, Díaz-Lazo $A$, Ubillús $M$, Aguí-Ortiz A, Rojas-Bravo V. Perception of knowledge and attitudes towards Covid-19 in a group of citizens from the urban Area of Huánuco. Rev Fac Med. Hum. 2021; 21(2):292-300. http://doi.org/10.25176/ rfmh.v21i1.3352

17. Bates BR, Tami A, Carvajal A, Grijalva MJ. Knowledge, attitudes, and practices towards COVID-19 among Venezuelans during the 2020 epidemic: An online cross-sectional survey. PLoS One. 2021; 16(4):e0249022. https://doi.org/10.1371/ journal.pone.0249022 
18. Bates BR, Moncayo AL, Costales JA, HerreraCespedes CA, Grijalva MJ. Knowledge, Attitudes, and Practices Towards COVID-19 Among Ecuadorians During the Outbreak: An Online Cross-Sectional Survey. J Community Health. 2020; 45(6):1158-1167. https:// doi.org/10.1007/s10900-020-00916-7

19. Rios C. Conocimientos, actitudes y prácticas hacia COVID-19 en paraguayos el periodo de brote: una encuesta rápida en línea. Rev. salud publica Parag. 2020; 10(2):1722. https://doi.org/10.18004/rspp. 2020. diciembre.17

20. Ngwewondo A, Nkengazong $L$, Ambe LA, Ebogo JT, Mba FM, Goni HO, et al. Knowledge, attitudes, practices of/towards COVID 19 preventive measures and symptoms: A cross-sectional study during the exponential rise of the outbreak in Cameroon. PLoS Negl Trop Dis. 2020; 14(9):e0008700. https:// doi.org/10.1371/journal.pntd.0008700

21. Sulistyawati S, Rokhmayanti R, Aji B, Wijayanti SPM, Hastuti SKW, Sukesi TW, et al. Knowledge, Attitudes, Practices and Information Needs During the COVID-19 Pandemic in Indonesia. Risk Manag Healthc Policy. 2021; 14:163-175. https://doi. org/10.2147/RMHP.S288579

22. Ferdous $M Z$, Islam MS, Sikder MT, Mosaddek ASM, Zegarra-Valdivia JA, Gozal D. Knowledge, attitude, and practice regarding COVID-19 outbreak in Bangladesh: An online-based cross-sectional study. PLoS One. $2020 ; 15(10):$ e0239254. https://doi. org/10.1371/journal.pone.0239254
23. Iqbal MA, Younas MZ. Public knowledge, attitudes, and practices towards COVID-19 in Pakistan: A cross-sectional study. Child Youth Serv Rev. 2021; 120:105784. https:// doi.org/10.1016/j.childyouth.2020.105784

24. Azlan AA, Hamzah MR, Sern TJ, Ayub SH, Mohamad E. Public knowledge, attitudes and practices towards COVID-19: A crosssectional study in Malaysia. PLoS One. 2020; 15(5):e0233668. https://doi.org/10.1371/ journal.pone. 0233668

25. Pignouli S. Escenarios sociales asociados con el brote de enfermedad por coronavirus (COVID-19). Astrolabio. 2020; 25:165-195. https://revistas.unc.edu.ar/index.php/ astrolabio/article/view/29420

26. Urrunaga-Pastor D, Bendezu-Quispe G, Herrera-Añazco P, Uyen-Cateriano A, ToroHuamanchumo CJ, Rodriguez-Morales $A J$, et al. Cross-sectional analysis of COVID-19 vaccine intention, perceptions and hesitancy across Latin America and the Caribbean. Travel Med Infect Dis. 2021; 41:102059. https://doi.org/10.1016/j. tmaid.2021.102059 